\title{
The Determination of all Imaginary, Quartic, Abelian Number Fields With Class Number 1
}

\author{
By Bennett Setzer
}

\begin{abstract}
In this paper, it is proved that there are just seven imaginary number fields, quartic cyclic over the rational field, and having class number 1 . These are the quartic, cyclic imaginary subfields of the cyclotomic fields generated by the $f$ th roots of unity, where $f$ is 16 or is a prime less than 100 . This completes the list of imaginary, quartic, abelian number fields with class number 1 . There are 54 such fields, with maximal conductor 67.163 .
\end{abstract}

In [5], Uchida proves the following about imaginary, quartic abelian number fields of class number 1 :

(A) If the field is bicyclic, there are 47 such fields, (with maximal conductor 67.163 ) with possibly one more.

(B) If the field is cyclic, the conductor must be less than 50000 .

The existence of another field in (A), depends, however, on there being an imaginary quadratic field with class number 2 and discriminant $<-427$. Stark [4], and Montgomery-Weinberger [3], have shown there is no such quadratic field, so Uchida's list of bicyclic fields is complete. Brown and Parry [1], using the results of Stark, have also given a complete list of imaginary bicyclic quartic fields of class number 1 .

This paper describes the computations that were carried out to show that there are just 7 imaginary, quartic cyclic number fields with class number 1 .

THEOREM. There are exactly 54 imaginary, quartic, abelian number fields with class number 1 :

(a) 47 bicyclic fields, with maximal conductor 67.163; and

(b) 7 cyclic fields, with maximal conductor 61 .

As will be seen below, the cyclic fields are contained in the cyclotomic fields $\Omega_{16}, \Omega_{5}, \Omega_{13}, \Omega_{29}, \Omega_{37}, \Omega_{53}$, and $\Omega_{61}$. $\left(\Omega_{N}\right.$ is the field generated by the $N$ th roots of unity.) Each is the unique imaginary, cyclic, quartic subfield of the corresponding cyclotomic field.

The theory of the relative class number developed by Hasse [2], was used to eliminate all possible fields up to the 50000 limit, except for the above seven. We describe the details relevant to the present problem. Let $k$ be an imaginary, cyclic quartic field. There is, associated to $k$, a pair of quartic Dirichlet characters $\psi$ and $\bar{\psi}$ of conductor $f=$ conductor of $k$. These are, essentially, the characters of order 4 on $\operatorname{Gal}\left(\Omega_{f} / Q\right) \stackrel{\sim}{\rightarrow}(Z / f)^{*}$, which are orthogonal to $\operatorname{Gal}\left(\Omega_{f} / k\right)$. Since $k$ is totally complex, 
$\psi(-1)=\bar{\psi}(-1)=-1$. Now, also, $k$ contains a unique real quadratic subfield, $k_{1}$. Let $h=$ class number of $k, h_{1}=$ class number of $k_{1}$. Then $h=h_{1} h^{*}$, where $h^{*}$ is an integer, the relative class number. Hasse (p. 79 of [2]), gives a formula for $h^{*}$, which in the present case reduces to

$$
h^{*}=w \Theta(\psi) \Theta(\bar{\psi}),
$$

where $w=$ number of roots of unity in $k$ and

$$
\Theta(\psi)=-\frac{1}{2 f} \sum_{x=1}^{f-1} x \psi(x) .
$$

(Note that it is easy to verify that a fundamental unit of $k_{1}$ is also a fundamental unit of $k$, so the factor $Q$ in Hasse's original formula is 1 in the present case.)

The search for $k$ of class number 1 was first reduced by the following

Proposition 1. If $h=1$, then $f=16$ or $f$ is a prime, $f \equiv 5(\bmod 8)$.

Proof. Since $h \geqslant h^{*} \geqslant 2 \Theta(\psi) \Theta(\bar{\psi})$, if $h$ is to be 1 , then $\Theta(\psi)$ cannot be integral. But, $\Theta(\psi)$ is integral if $f$ is divisible by two distinct primes; Section 28 of [2]. If $f=p^{e}$ is a power of an odd prime, then $\Theta(\psi)$ is integral if $e>1$; Satz 32, p. 93 of [2]. For there to be a quartic character $(\bmod f), f$ an odd prime, we must have $f \equiv 1(\bmod 4)$. If the character is to be odd, then $f \neq 1(\bmod 8)$. Finally, if $f=2^{e}$, then $f=16$, since the only odd quartic characters $\left(\bmod 2^{e}\right)$ have conductor 16 .

In each case $f=16, f$ a prime $\equiv 5(\bmod 8)$, the field $k$ is a uniquely determined subfield of $\Omega_{f}$, and the character pair is the unique pair of odd, quartic characters $(\bmod f)$. For $f=16, k_{1}=Q[\sqrt{2}]$ so $h_{1}=1$. Also, $w=2$. It is easily checked that $\Theta(\psi) \Theta(\bar{\psi})=1 / 2$. So, in the case $f=16, h=1$. Now assume that $f$ is a prime $\equiv 5$ $(\bmod 8)$. The computation of $\Theta(\psi)$ can be done by counting quartic residues $(\bmod f)$.

PROPOSITION 2. Let $f$ be a prime $\equiv 5(\bmod 8)$ and $\psi$ a quartic character $(\bmod f)$, where $\psi(2)=i, i^{2}=-1$. Then

$$
\Theta(\psi)=\left(\left(3\left(\alpha_{0}-\delta_{0}\right)+\left(\beta_{0}-\gamma_{0}\right)\right)+\left(\left(\alpha_{0}-\delta_{0}\right)-3\left(\beta_{0}-\gamma_{0}\right)\right) i\right) / 10,
$$

and

$$
\Theta(\psi) \Theta(\bar{\psi})=\left(\left(\alpha_{0}-\delta_{0}\right)^{2}+\left(\beta_{0}-\gamma_{0}\right)^{2}\right) / 10
$$

where $\alpha_{0}$ is the number of quartic residues $(\bmod f)$ contained in the interval $[1,(f-1) / 4] ; \beta_{0}$, in $[(f+3) / 4,(f-1) / 2] ; \gamma_{0}$, in $[(f+1) / 2,(3 f-3) / 4] ; \delta_{0}$, in $[(3 f+1) / 4,(f-1)]$.

Note that, since $f \equiv 5(\bmod 8)$ is assumed, 2 is not a quadratic residue $(\bmod f)$, so $(\psi(2))^{2}=-1$.

Note also that the definition of $\Theta(\psi)$ and Proposition 2 give $\Theta(\psi)$ as members of the Gaussian field $(Q[i])$ with denominators $2 f$ and 10 , respectively. If $f>5$, the true denominator is at worst 2 , so $3\left(\alpha_{0}-\delta_{0}\right)+\left(\beta_{0}-\gamma_{0}\right) \equiv 0(\bmod 5)$. Also, $\alpha_{0}+$ $\beta_{0}+\gamma_{0}+\delta_{0}=(f-1) / 4 \equiv 1(\bmod 2)$, so $\Theta(\psi)$ and $\Theta(\psi) \cdot \Theta(\bar{\psi})$ have exact denominator 2. This also follows from Satz 32 of [2]. 
Proof. We first derive the formula

$$
\Theta(\psi)=\psi(2) \sum_{x=1}^{(f-1) / 2} \psi(x) /(4 \psi(2)-2)
$$

Let

$$
G_{1}=\sum_{x=1}^{(f-1) / 2} x \psi(x), \quad G_{2}=\sum_{x=(f+1) / 2}^{f-1} x \psi(x)
$$

Then

$$
2 \psi(2) G_{1}=\sum_{x=1}^{(f-1) / 2} 2 x \psi(2 x)
$$

and

$$
2 \psi(2) G_{2}=\sum_{x=(f+1) / 2}^{f-1}(2 x-f) \psi(2 x-f)-f \psi(2) \sum_{x=1}^{(f-1) / 2} \psi(x) .
$$

Since $f$ is odd,

$$
2 \psi(2)\left(G_{1}+G_{2}\right)=G_{1}+G_{2}-f \psi(2) \sum_{x=1}^{(f-1) / 2} \psi(x) .
$$

As $\Theta(\psi)=\left(G_{1}+G_{2}\right) /(-2 f),(1)$ is proved.

Now, the subgroup of quartic residues $(\bmod f)$ is index 4 in $(Z / f Z)^{*}$, with coset representatives $1,2,4,8$. Let $\alpha_{j}$ be the number of elements in $2^{j}$-coset contained in the interval $[1,(f-1) / 4]$, for $0 \leqslant j \leqslant 3$. ( $\alpha_{0}$ is the same as above.) Define $\beta_{j}, \gamma_{j}, \delta_{j}$ similarly for the successive quarter intervals of $[1, f-1]$. Then

$$
\sum_{x=1}^{(f-1) / 2} \psi(x)=\left(\alpha_{0}+\beta_{0}\right)-\left(\alpha_{2}+\beta_{2}\right)+\left(\alpha_{1}+\beta_{1}\right) i-\left(\alpha_{3}+\beta_{3}\right) i .
$$

Since -1 is a quadratic, but not quartic, residue $(\bmod f)$, multiplication by -1 gives the following relations:

$$
\begin{array}{llll}
\alpha_{0}=\delta_{2}, & \alpha_{1}=\delta_{3}, & \alpha_{2}=\delta_{0}, & \alpha_{3}=\delta_{1}, \\
\beta_{0}=\gamma_{2}, & \beta_{1}=\gamma_{3}, & \beta_{2}=\gamma_{0}, & \beta_{3}=\gamma_{1} .
\end{array}
$$

Also, the quartic residues in $[1,(f-1) / 4]$ and $[(f+1) / 2,(3 f-3) / 4]$, when multiplied by 2 , map one to one onto the 2-coset elements in $[1,(f-1) / 2]$. Thus $\alpha_{0}+\gamma_{0}=$ $\alpha_{1}+\beta_{1}$. Similarly:

$$
\begin{array}{ll}
\alpha_{0}+\gamma_{0}=\alpha_{1}+\beta_{1}, & \beta_{0}+\delta_{0}=\gamma_{1}+\delta_{1}, \\
\alpha_{1}+\gamma_{1}=\alpha_{2}+\beta_{2}, & \beta_{1}+\delta_{1}=\gamma_{2}+\delta_{2}, \\
\alpha_{2}+\gamma_{2}=\alpha_{3}+\beta_{3}, & \beta_{2}+\delta_{2}=\gamma_{3}+\delta_{3}, \\
\alpha_{3}+\gamma_{3}=\alpha_{0}+\beta_{0}, & \beta_{3}+\delta_{3}=\gamma_{0}+\delta_{0} .
\end{array}
$$


(3) and (4) imply

$$
\begin{array}{ll}
\alpha_{0}=\beta_{1}=\gamma_{3}=\delta_{2}, & \alpha_{2}=\beta_{3}=\gamma_{1}=\delta_{0}, \\
\alpha_{1}=\beta_{2}=\gamma_{0}=\delta_{3}, & \alpha_{3}=\beta_{0}=\gamma_{2}=\delta_{1} .
\end{array}
$$

Combining (5), (2) and (1), then rationalizing the denominator $(4 \psi(2)-2=$ $4 i-2)$, proves the proposition.

A program was written to compute $\Theta(\psi)$ for prime $f \equiv 5(\bmod 8), f \leqslant 50021$. The computation was done on an IBM 370. A machine language subroutine was used to compute, for each $f, x^{4}(\bmod f)$ for $1 \leqslant x \leqslant(f-1) / 2$. The count of $\alpha_{0}, \beta_{0}, \gamma_{0}$, $\delta_{0}$ was made from this. The whole computation took less than 10 minutes of CPU time. For $h=1, \Theta(\psi) \cdot \Theta(\bar{\psi})$ must be $1 / 2$ or less. This occurred only for $f=5$ (for which $\Theta(\psi) \Theta(\bar{\psi})=1 / 10$ ) and $f=13,29,37,53,61$ (for which $\Theta(\psi) \Theta(\bar{\psi})=1 / 2$ ).

Proof of Theorem. By Uchida's result, it only remains to show that the seven cyclic fields do have class number 1 . The case $f=16$ was considered before. For $f=5$, $k=\Omega_{5}$ and $k_{1}=Q[\sqrt{5}]$. So, $w=10, h_{1}=1$ and $\Theta(\psi) \Theta(\bar{\psi})=1 / 10$, that is, $h=1$. In the remaining five cases, $k_{1}=Q[\sqrt{f}]$ and $h_{1}=1$. Also, $w=2, \Theta(\psi) \Theta(\bar{\psi})=1 / 2$ so $h=1$ in these cases.

Generating equations for these fields can be obtained by observing that $k=$ $k_{1}[\sqrt{\pi}]$, where $\pi=-\sqrt{f} \epsilon(-\sqrt{2} \epsilon$ for $f=16)$ and $\epsilon>0$ is a fundamental unit for $k_{1}$ (norm of $\epsilon$ is -1 , necessarily). Then one has

$$
\begin{aligned}
& f=5, \quad x^{4}+5 x^{2}+5=0, \\
& f=13, \quad x^{4}+13 x^{2}+13=0, \\
& f=29, \quad x^{4}+29 x^{2}+29=0 \text {, } \\
& f=37, \quad x^{4}+74 x^{2}+37=0, \\
& f=53, \quad x^{4}+53 x^{2}+53=0, \\
& f=61, \quad x^{4}+305 x^{2}+61=0, \\
& f=16, \quad x^{4}+4 x^{2}+2=0 \text {. }
\end{aligned}
$$

The computer resources used in this paper were provided by the Computer Center at the University of Illinois, Chicago Circle.

\section{Mathematics Department \\ University of Illinois at Chicago Circle \\ Chicago, Illinois 60680}

1. E. BROWN \& C. J. PARRY, "The imaginary bicyclic biquadratic fields with class number 1,"J. Reine Angew. Math., v. 266, 1974, pp. 118-120.

2. HELMUT HASSE, Über die Klassenzahl abelscher Zahlkorper, Akademie-Verlag, Berlin, 1952.

3. H. L. MONTGOMERY \& P. J. WEINBERGER, "Notes on small class numbers," Acta Arith., v. 24, 1974, pp. 529-542.

4. HAROLD STARK, "On complex quadratic fields with class number two," Math. Comp., v. 29, 1975, pp. 289-302.

5. KÔJI UCHIDA, "Imaginary abelian number fields with class number 1," Təhoku Math. J. (2), v. 24, 1972 , pp. 487-499. 